Supplement of Biogeosciences, 14, 177-186, 2017

http://www.biogeosciences.net/14/177/2017/

doi:10.5194/bg-14-177-2017-supplement

(C) Author(s) 2017. CC Attribution 3.0 License.

(c) (i)

Supplement of

\title{
Role of zooplankton in determining the efficiency of the biological carbon pump
}

Emma L. Cavan et al.

Correspondence to: Emma L. Cavan (emma.cavan@utas.edu.au)

The copyright of individual parts of the supplement might differ from the CC-BY 3.0 licence. 
1 Table S1. Site information including the depth the MSCs were deployed to.

\begin{tabular}{|c|c|c|c|c|c|}
\hline Station \# & Site & Latitude $\left({ }^{\circ} \mathrm{N}\right)$ & Longitude $\left({ }^{\circ} \mathrm{W}\right)$ & Date & MSC depth (m) \\
\hline 1 & $\mathrm{SO}$ & -59.9096 & 53.03006 & $15 / 1 / 2013$ & 25,125 \\
\hline 2 & SO & -58.51637 & 51.25974 & $16 / 1 / 2013$ & 40,140 \\
\hline 3 & $\mathrm{SO}$ & -59.91357 & 49.41599 & $17 / 1 / 2013$ & 40,140 \\
\hline 4 & $\mathrm{SO}$ & -60.97059 & 48.13545 & $18 / 1 / 2013$ & 40,140 \\
\hline 5 & SO & -57.97091 & 42.93436 & $20 / 1 / 2013$ & 40,140 \\
\hline 6 & $\mathrm{SO}$ & -55.20345 & 41.32185 & $21 / 1 / 2013$ & 40,140 \\
\hline 7 & $\mathrm{SO}$ & -52.90708 & 40.13592 & $22 / 1 / 2013$ & 40,140 \\
\hline 8 & $\mathrm{SO}$ & -50.28753 & 39.02036 & $23 / 1 / 2013$ & 40,140 \\
\hline 9 & $\mathrm{SO}$ & -50.95719 & 37.61516 & $24 / 1 / 2013$ & 70,170 \\
\hline 10 & SO & -52.68932 & 36.62300 & $25 / 1 / 2013$ & 70,170 \\
\hline 11 & $\mathrm{SO}$ & -51.68808 & 34.97653 & $28 / 1 / 2013$ & 70,170 \\
\hline 12 & $\mathrm{SO}$ & -51.36901 & 31.09135 & $29 / 1 / 2013$ & 70,170 \\
\hline 13 & $\mathrm{SO}$ & -53.6265 & 29.32742 & $30 / 1 / 2013$ & 70,170 \\
\hline 14 & $\mathrm{SO}$ & -55.92838 & 27.25843 & $31 / 1 / 2013$ & 70,170 \\
\hline 15 & SO & -58.08564 & 25.92572 & $1 / 2 / 2013$ & 70,170 \\
\hline 16 & $\mathrm{SO}$ & -63.25913 & 25.35395 & $3 / 2 / 2013$ & 20,120 \\
\hline 17 & SO & -62.26828 & 26.84829 & $4 / 2 / 2013$ & 70,170 \\
\hline 18 & $\mathrm{SO}$ & -60.14215 & 29.48655 & $5 / 2 / 2013$ & 70,170 \\
\hline 19 & PAP & 48.3869 & 16.08420 & $4 / 6 / 2013$ & 60,160 \\
\hline 20 & PAP & 48.3892 & 16.08580 & $5 / 6 / 2013$ & 40,140 \\
\hline 21 & PAP & 48.3891 & 16.08570 & $7 / 6 / 2013$ & 45,145 \\
\hline 22 & PAP & 48.3892 & 16.08750 & $7 / 6 / 2013$ & 45,145 \\
\hline 23 & PAP & 48.3894 & 16.08580 & $9 / 6 / 2013$ & 45,145 \\
\hline 24 & ETNP & 13.2464 & 91.22750 & $15 / 1 / 2014$ & 40,120 \\
\hline 25 & ETNP & 13.2129 & 91.22780 & $21 / 1 / 2014$ & 70,220 \\
\hline 26 & ETNP & 13.1370 & 91.17910 & $27 / 1 / 2014$ & 40,120 \\
\hline 27 & ETNP & 13.0418 & 91.11520 & $2 / 2 / 2014$ & 50,110 \\
\hline
\end{tabular}


5 Table S2. Fast and slow sinking mean $b$ and $z *$ at each site. NA shows sites where the range

6 was too large $(>1000)$ and $n$ too small $(<5)$ to produce an accurate mean. ' - ' depicts where

7 mean $z^{*}$ values where negative because of higher fluxes at depth, suggesting POC fluxes

8 going into the atmosphere.

\begin{tabular}{cccccc}
\hline & \multicolumn{2}{c}{$\boldsymbol{b}$} & \multicolumn{2}{c}{$\boldsymbol{z}^{*}(\mathrm{~m})$} \\
\hline Sinking & Site & Model & Observation & Model & Observation \\
\hline Fast & SO & 0.63 & 0.47 & 229 & - \\
& PAP & 0.72 & 0.57 & 199 & 329 \\
& ETNP & 0.70 & 0.24 & 207 & - \\
Slow & SO & 0.87 & 1.13 & 166 & - \\
& PAP & 1.27 & NA & 113 & 169 \\
& ETNP & 1.47 & NA & 98 & 33 \\
\hline
\end{tabular}



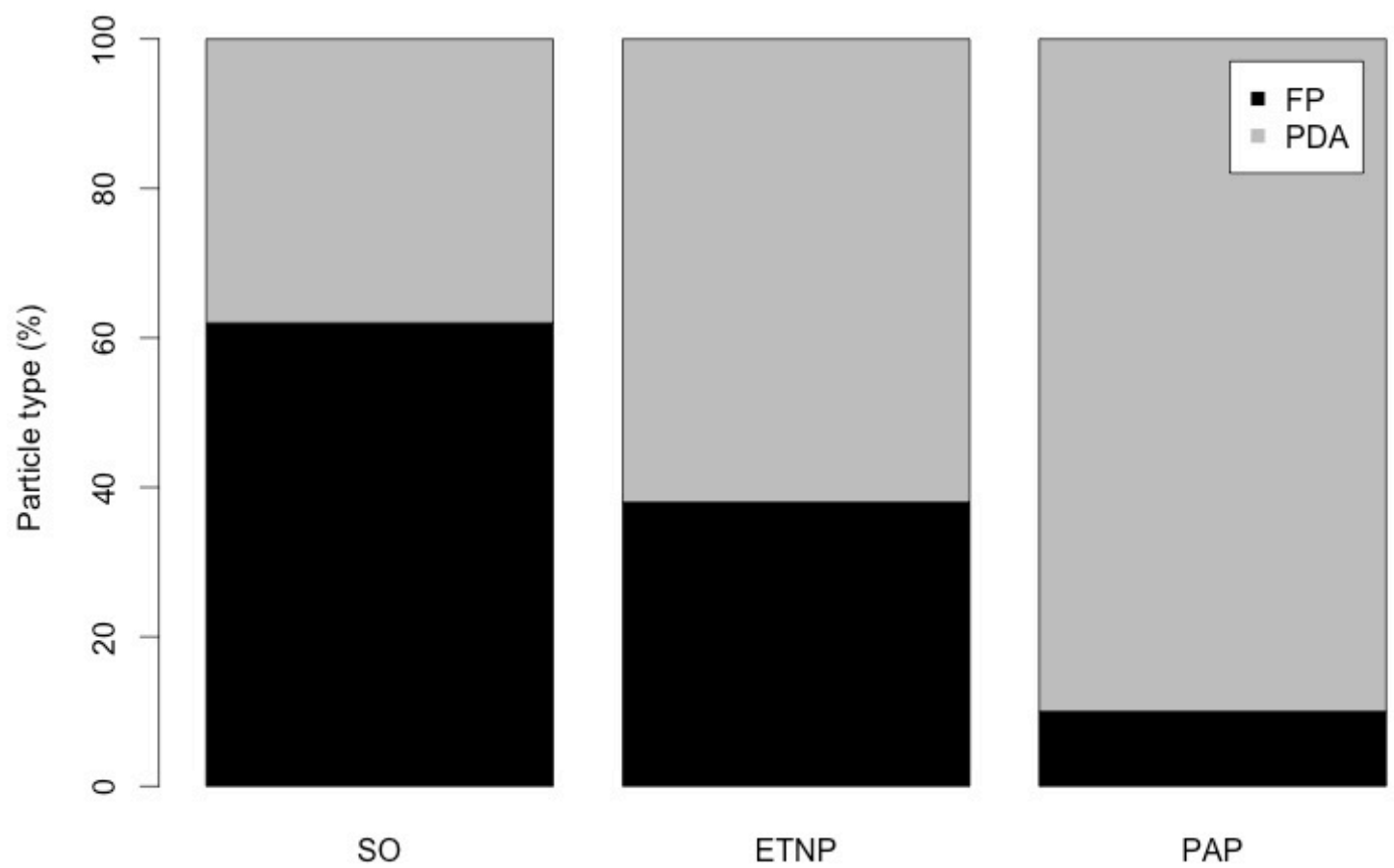

36 Figure S1. Abundance of phytodetrital aggregates (PDAs) and faecal pellets (FPs) as

37 percentage of total particle flux. FPs dominated in the SO but PDAs were most abundant in 38 both the ETNP and at PAP.

39

40

41

42

43

44

45

46

47

48 
(a)

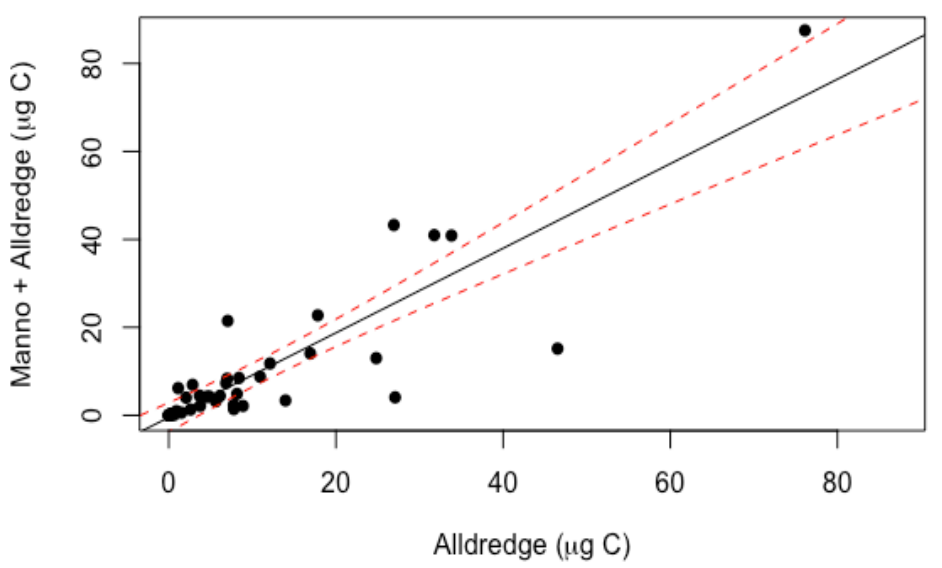

(c)

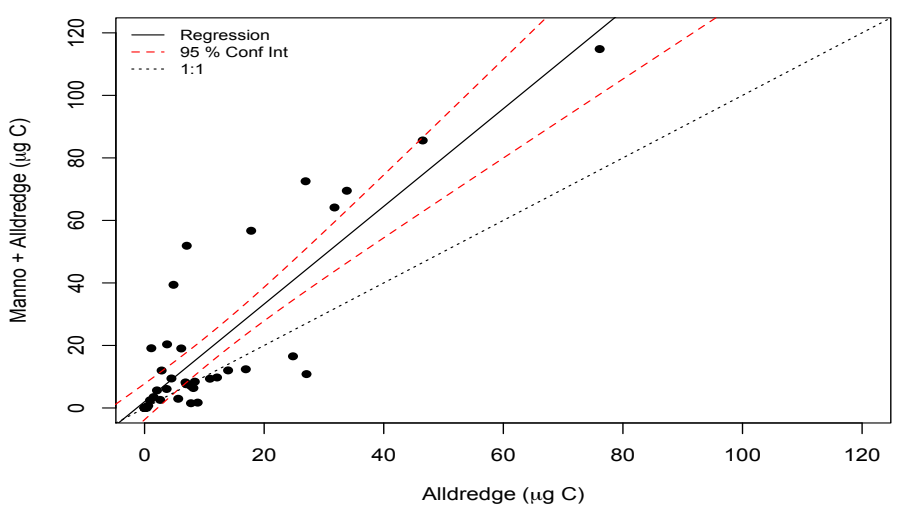

49

52 Figure S2. Conversion factor (CF) sensitivity analysis using Southern Ocean observational

53 data. Comparison of total (phytodetrital aggregate + faecal pellet) fast sinking mass for MSC

54 deployment using Alldredge CFs for both PDAs and FPs (x-axis) and Alldredge CFs for PDAs and Manno CFs for FPs (y-axis) in winter (a) and summer (c). Solid black line is the

56 regression (slope 0.96 ) and dashed red lines the $95 \%$ confidence intervals. Note FPs

57 dominated particle abundance in the Southern Ocean (see Fig. S3). b) Primary production against particle export efficiency using masses in a) and c), winter (b) and summer (d). 

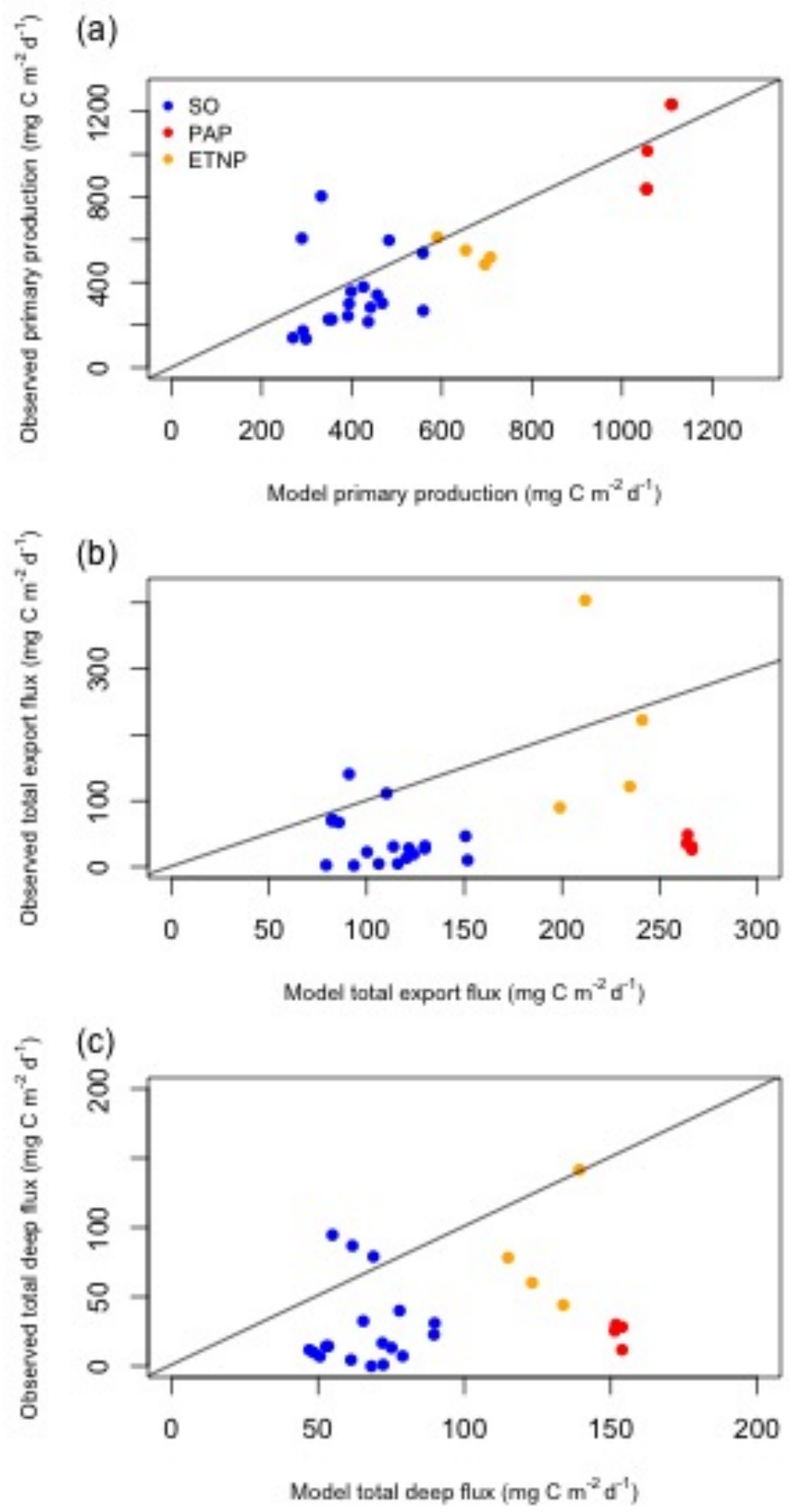

61 Fig. S3. Comparison of observations and model outputs for (a) primary production, (b) total

62 sinking export and (c) deep fluxes with the 1:1 line (black). Blue points are Southern Ocean,

63 red PAP and orange equatorial Pacific. 

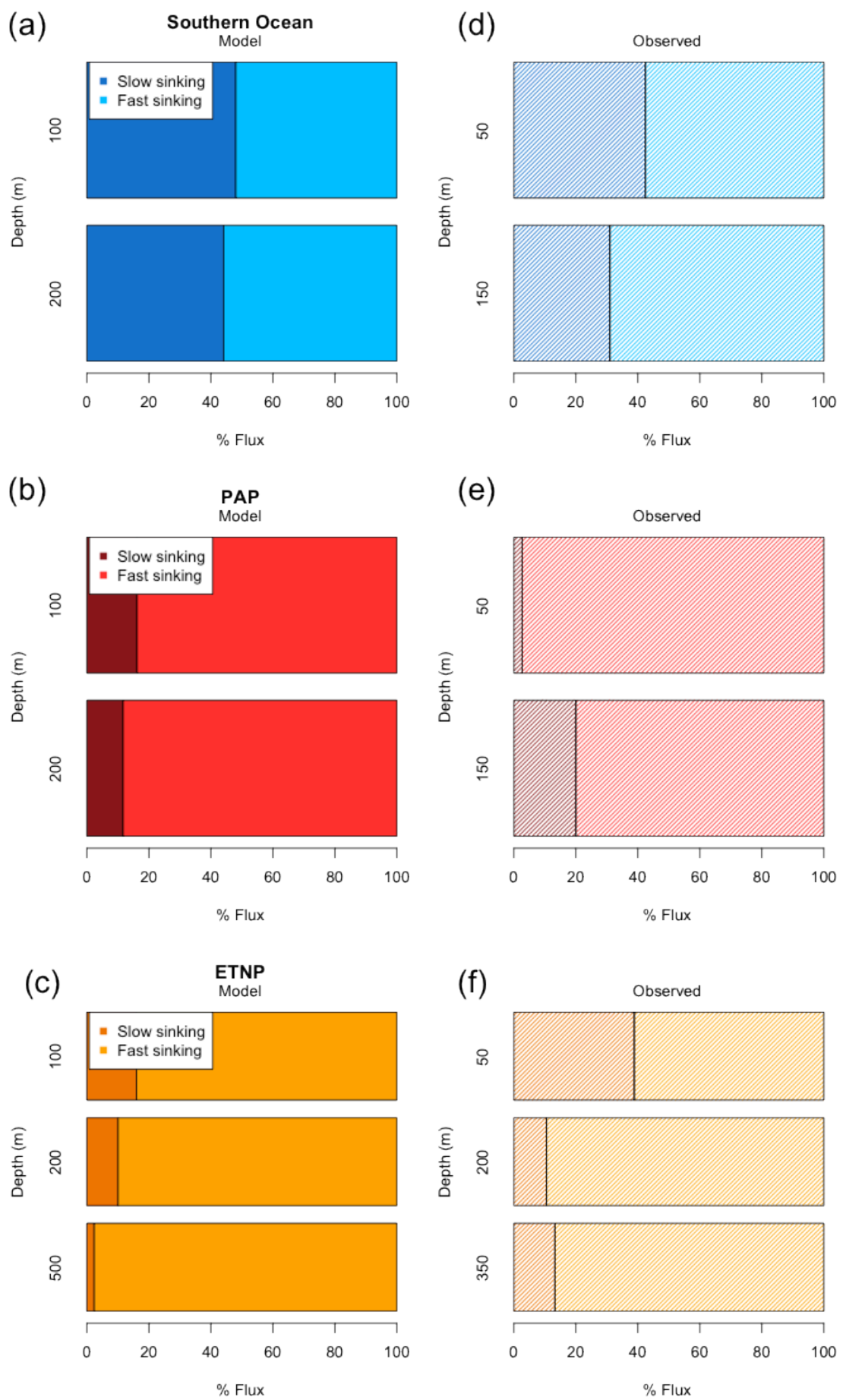

64

65 Fig. S4. Percentage contribution of fast and slow sinking fluxes to total sinking fluxes from

66 the model (a-c) and observations (d-f) at all site, SO (a \& d), PAP (b \& e) and the ETNP (c \&

67 f). Slow sinking fluxes are the darker shading on each plot. 